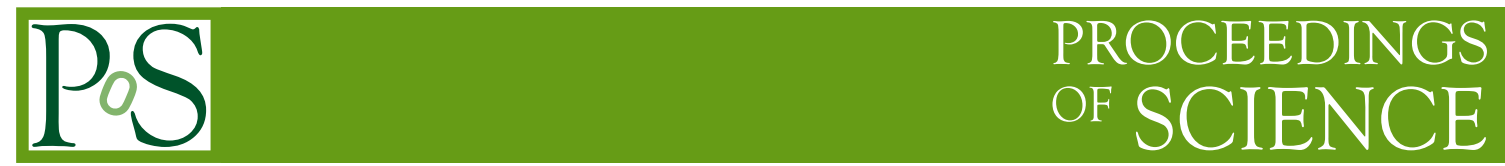

\title{
Universality and Scaling at the chiral transition in two-flavor QCD at finite temperature
}

\author{
Tereza Mendes \\ Instituto de Física de São Carlos, Universidade de São Paulo, \\ Caixa Postal 369, 13560-970 São Carlos, SP, Brazil \\ E-mail: mendes@ifsc.usp.br
}

\begin{abstract}
The order of the phase transition in finite-temperature QCD with two degenerate light quarks is still an open problem and corresponds to the last question mark in the zero-density phase diagram of QCD. We argue that establishing the nature of the transition in this case is also a crucial test for numerical simulations of lattice QCD, allowing precise estimates of possible systematic errors related e.g. to the choice of fermion-simulation algorithm or of discretized formulation for fermions.
\end{abstract}

The XXV International Symposium on Lattice Field Theory

July 30-4 August 2007

Regensburg, Germany 


\section{Introduction}

The phase diagram at zero baryon density and varying quark masses has been intensively investigated by lattice simulations [1]. The case of two dynamic quarks, i.e. considering dynamic effects of only two degenerate light-quark flavors, corresponding to the up and down quarks, is particularly interesting. In this case, if the transition is of second order, one would expect to observe universal critical scaling in the class of the $3 d O(4)$ continuous-spin model [2]. Also, in the continuum limit, simulations using different discretizations for the fermion fields should give the same results. The fact that the critical behavior should be in the universality class of a spin model can be precisely checked, since the nonperturbative behavior for these models can be obtained with Monte Carlo simulations by so-called global methods, which avoid the critical slowing-down present in QCD simulations.

The determination of the correct nature of the transition in the two-flavor case is one of the present challenges of lattice QCD. In fact, it corresponds to the last question mark in the zerodensity phase diagram (see e.g. [1]). This prediction has been investigated numerically by lattice simulations for almost twenty years, yet there is still no agreement about the order of the transition or about its scaling properties. More precisely, the predicted $O(4)$ scaling has been observed in the Wilson-fermion case [4], but not in the staggered-fermion case, believed to be the appropriate formulation for studies of the chiral region. In this case, extensive numerical studies and scaling tests have been done in the past by the Bielefeld [5], JLQCD [6] and MILC [7] groups. It was found that the chiral-susceptibility peaks scale reasonably well with the predicted exponents, but no agreement is seen in a comparison with the $O(4)$ scaling function. At the same time, some recent numerical studies with staggered fermions claim that the deconfining transition may be of first order $[8,9]$. On the other hand, studies of the two-flavor case in the massless limit point towards a second-order transition in the $O(2)$ universality class [10]. In summary, the issue of the nature of the QCD transition for the two-flavor zero-density case is still an open problem, just as it was ten years ago [11].

Although scaling is not observed, a simple method may be used [12, 13] in order to obtain a uniquely defined normalization of the QCD data, allowing an unambiguous comparison to the (normalized) $O(4)$ scaling function. This analysis shows a surprisingly better agreement for the larger values of the quark masses. Let us note that in previous scaling tests the comparison had been done up to a (non-universal) normalization of the data and a match to the scaling function was tried by fitting it to the data points with the smallest masses. One interpretation of this result is that data at smaller masses (closer to the physical values) suffer more strongly from systematic errors in the simulations. In fact, larger quark masses are much easier to simulate, allowing greater control over errors and more reliable results. Here we present a study at a rather large value for the light quark mass $m_{q}$ (we take $m_{q}=0.075$ in lattice units), using staggered fermions and the MILC code. We consider the standard (i.e., unimproved) action and temporal lattice extent $N_{\tau}=4$, as in most of the studies mentioned above. Also, we use the so-called $\mathrm{R}$ algorithm, but consider very small integration steps (of about $m_{q} / 10$ ). 


\section{Scaling tests}

The behavior of systems around a second-order phase transition (or critical point) may show striking similarities for systems that would otherwise seem completely different. In fact, it is possible to divide systems into so-called universality classes, in such a way that each class will have, e.g., the same critical exponents around the transition. The critical point corresponds to external magnetic field $H$ equal to zero, and temperature $T$ given by its critical value $T_{c}$. Typical exponents are

$$
\begin{aligned}
M_{h=0, t \rightarrow 0^{-}} & \rightarrow|t|^{\beta}, \\
\chi_{h=0, t \rightarrow 0} & \rightarrow|t|^{-\gamma}, \\
M_{t=0, h \rightarrow 0} & \rightarrow h^{1 / \delta},
\end{aligned}
$$

where $M$ is the order parameter - e.g. the magnetization for a spin system $-\chi$ is the corresponding susceptibility and $t=\left(T-T_{c}\right) / T_{0}, h=H / H_{0}$ are the reduced temperature and magnetic field, respectively. ( $T_{0}$ and $H_{0}$ are normalization constants.)

Thus, in principle, one may compare the critical exponents for different systems to check if they belong to the same universality class. In practice, however, the critical exponents may vary little from one class to the other and in order to carry out the comparison one would need to have a very precise determination of the exponents, which is not yet feasible in the QCD case.

A more general comparison is obtained through the scaling functions for both systems. This comparison allows a more conclusive test, and can be applied for cases where the critical exponents cannot be established with great accuracy. In this case we may assume the exponents for a given class and compare the behavior of the whole critical region for one system to the known scaling curve for the proposed universality class. The scaling Ansatz is written for the free energy $F_{s}$ in the critical region as

$$
F_{s}(t, h)=b^{-d} F_{s}\left(b^{y_{t}} t, b^{y_{h}} h\right),
$$

where $b$ is a rescaling factor, $d$ is the dimension and $y_{t}, y_{h}$ are related to the usual critical exponents: $\beta, \gamma, \delta$ mentioned above. Correspondingly, the order parameter must be described by a universal function

$$
M / h^{1 / \delta}=f_{M}\left(t / h^{1 / \beta \delta}\right) .
$$

The statement that the function $f_{M}$ is universal means that once the non-universal normalization constants $T_{0}$ and $H_{0}$ are determined for a given system in the universality class, the order parameter $M$ scales according to the same scaling function $f_{M}$ for all systems in this class. As said above, the comparison of (normalized) scaling functions between two systems is a more general test of universality, especially in the case of the QCD phase transition.

A further difficulty in studying the critical behavior at the QCD phase transition is the impossibility of considering the critical point directly, since that would correspond to having zero quark mass, or zero magnetic field $H$ in the language of the spin models above. In order to check scaling with critical exponents of a given class, or to determine the normalization constants $T_{0}$ and $H_{0}$ for systems where a study at $H=0$ is not possible, it is important to determine the pseudo-critical line, defined by the points where the susceptibility $\chi$ shows a (finite) peak. This corresponds to the 
rounding of the divergence that would be observed for $H=0, T=T_{c}$. The susceptibility scales as

$$
\chi=\partial M / \partial H=\left(1 / H_{0}\right) h^{1 / \delta-1} f_{\chi}\left(t / h^{1 / \beta}\right),
$$

where $f_{\chi}$ is a universal function related to $f_{M}$. At each fixed $h$ the peak in $\chi$ is given by

$$
\begin{aligned}
t_{p} & =z_{p} h^{1 / \beta \delta}, \\
M_{p} & =h^{1 / \delta} f_{M}\left(z_{p}\right), \\
H_{0} \chi_{p} & =h^{1 / \delta-1} f_{\chi}\left(z_{p}\right) .
\end{aligned}
$$

Thus, the behavior along the pseudo-critical line is determined by the universal constants $z_{p}$, $f_{M}\left(z_{p}\right), f_{\chi}\left(z_{p}\right)$. Critical exponents, the scaling function $f_{M}$ and the universal constants above are well-known for the $3 d O(4)$ model $[14,15,16]$.

Note that one may also consider the comparison for finite-size-scaling functions, since they are also universal and have the advantage of being valid for finite values of $L$, the linear size of the system. Such functions can be determined numerically [15] for the $3 d O(4)$ model.

\section{Comparison of QCD data with the predicted scaling function}

We now turn to the comparison of the two-flavor QCD data in the critical region (in the case of small but nonzero quark mass) to the predicted scaling properties of the $3 d O(4)$ spin model. As mentioned in the Introduction, we consider the chiral phase transition, since there is no clear order parameter for the deconfinement transition in the case of full QCD. The order parameter for the chiral transition is given by the so-called chiral condensate $\langle\bar{\psi} \psi\rangle$, where $\psi$ is a combination of the quark fields entering the QCD Lagrangian. The analogue of the magnetic field is the quark mass $m_{q}$, and (on the lattice) the reduced temperature is proportional to $6 / g^{2}-6 / g_{c}^{2}(0)$, where $g$ is the lattice bare coupling and $g_{c}^{2}(0)$ is its extrapolated critical value. Therefore, referring to the pseudo-critical line described in the previous section, the chiral susceptibility peaks at

$$
t_{p} \sim m_{q}^{1 / \beta \delta}
$$

As mentioned in the Introduction, previous results from lattice-QCD simulations in the twoflavor case show good scaling (with the predicted exponents) only along the pseudo-critical line, which is given by the peaks of the chiral susceptibility. It should be clear from the discussion in the above sections that this is not a sufficient test to prove that the transition is second order, especially if no agreement is seen when comparing the data to the scaling function. We use [13] the observed scaling along the pseudo-critical line and the universal quantities $z_{p}, f_{M}\left(z_{p}\right)$ from the $O(4)$ model to determine the normalization constants $H_{0}, T_{0}$ for the QCD data. This allows an unambiguous comparison of the data to the scaling function $f_{M}$. More precisely, we note that in previous analyses the normalization constants were tentatively adjusted by shifting the $O(4)$ curve so as to get a rough agreement with the data at smaller quark masses, since these are closer to the chiral limit. The problem is that the lighter masses are also more subject to the presence of systematic errors in the simulations. In this case the overall agreement was rather poor, indicating that there were strong systematic effets or that the transition is not in the predicted universality class. Here we fix the 
constants as described in Section 2, following the behavior along the pseudo-critical line. In this way no value of the quark mass is priviledged and the comparison is unambiguous.

Our comparison [13] is shown in Fig. 1 below. We note that the integration step for the data at the largest mass is considerably smaller than the usual values. (Our runs were done with 7000 trajectories and 125 steps of length 0.008 per trajectory.) The pseudo-critical line corresponds to a single point in this plot and is marked with an arrow. For clarity we do not show the data - from the Bielefeld and JLQCD collaborations - obtained directly at the pseudo-critical point. These are slightly scattered around $z_{p}$ but show good scaling within errors.

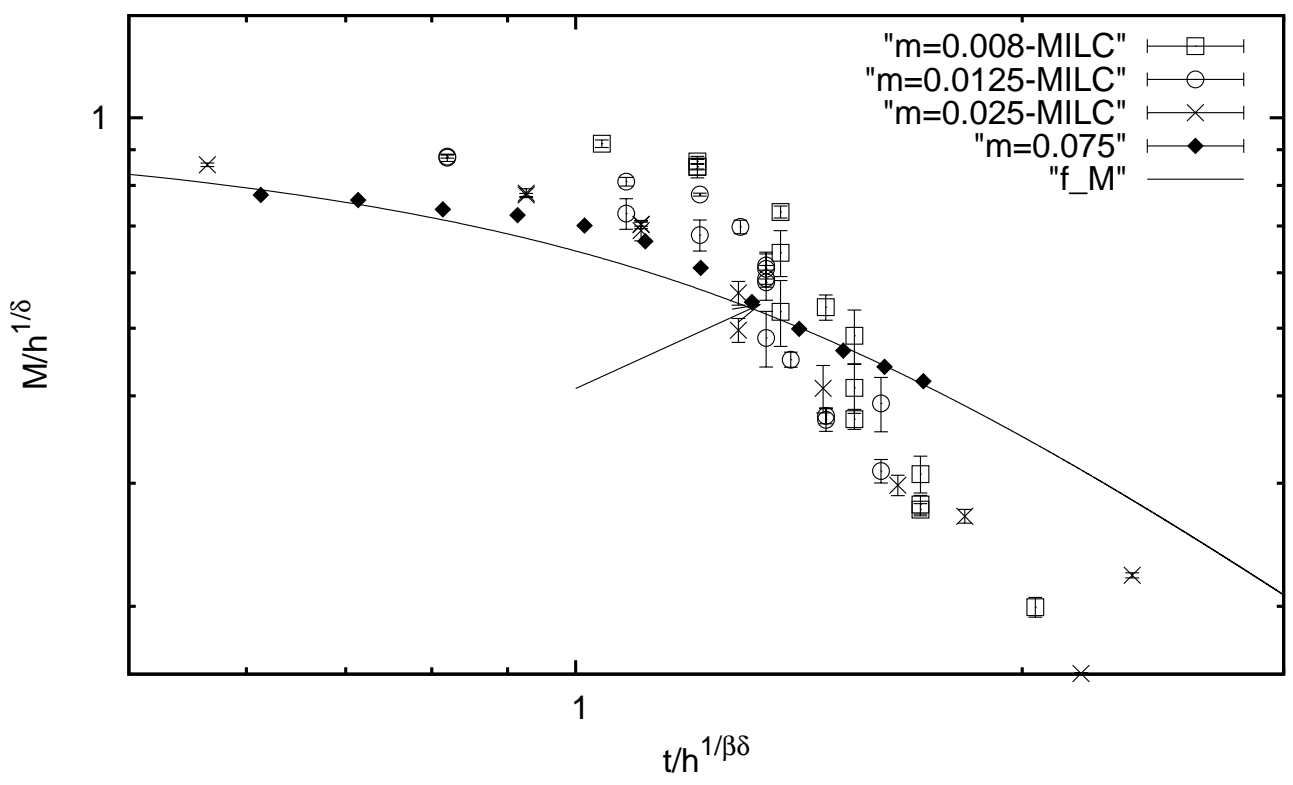

Figure 1: Comparison of QCD (staggered) data to the $O(4)$ scaling function [13]. For clarity, we do not show the data around the pseudo-critical point (indicated by the arrow), which were used to determine the normalization of the remaining data points.

We see relatively good scaling in the pseudo-critical region, i.e. around $\left[z_{p}, f_{M}\left(z_{p}\right)\right]$, as expected. Away from this region most MILC points are several standard deviations away from the predicted curve. These data are given for three values of the quark mass in lattice units: 0.008 , 0.0125 and 0.025 . Note that the points with larger mass come closer to the curve. In particular, we can see that the new data at $m_{q}=0.075$ show sensibly better scaling, especially for larger temperatures, where previously the scaling seemed unlikely. The good agreement of these data with the $O(4)$ scaling function motivates a careful study of systematic errors for smaller masses. A possible source of such errors are finite-size corrections, which would be stronger for smaller masses, since then the lattice side may not be large enough to "contain" the physical particle. Put differently, finite-size effects are expected when the correlation length (in lattice units) associated with a particle is comparable to or larger than the lattice side. Of course, this is more likely to occur for a lighter particle.

We note that a finite-size-scaling analysis may also be carried out using universal functions from the $O(4)$ case (see [13] and references thereof), but one finds that the QCD data show good (finite-size) scaling only along the pseudo-critical line. Moreover, with the temperature and mass 
parameters considered, the QCD data lie within the asymptotic region, where infinite-volume behavior should already be seen. In any case, the finite-size scaling away from the pseudo-critical region is also significantly better for the larger quark masses.

\section{Conclusions}

Determining the nature of the chiral phase transition in two-flavor QCD still stands as a challenge. Despite great computational effort, the prediction of a second-order transition with critical behavior in the universality class of the $O(4)$ spin model is not verified for staggered fermions of small masses, although it can be shown (by an unambiguous normalization of the data) that better scaling is obtained for the existing data at larger (unphysical) masses. Let us also mention that a redefinition of the reduced temperature in terms of the physical temperature $T$ including [8] a term in the quark mass $m_{q}$, improves the agreement with the scaling curve further [12].

The fact that data for heavier quarks would show such good scaling may be surprising, since the normalization of the data for comparison with the scaling curve did not priviledge any particular values of the quark mass. Indeed, one would expect to observe agreement with the $O(4)$ scaling curve for the smaller masses, which are closer to the chiral limit, where universality is predicted to hold. This suggests that the lack of scaling at small masses observed so far may be caused by systematic effects. A common source of systematic errors in lattice simulations are discretization effects, which might be related here to the small number of points along the temporal direction $\left(N_{\tau}=4\right)$ or to the use of the unimproved fermion action. The use of the staggered fermion formulation has also been generally criticized recently [17]. Other sources of errors could be finite-size corrections and uncontrolled errors in the hybrid Monte Carlo algorithm (the R algorithm, in our case) used for updating the configurations. Both these sources of errors would be more significant for the case of smaller masses.

We note that, as mentioned above, the deviations from $O(4)$ scaling at smaller masses are most likely not due to finite-size corrections. On the other hand, it must be stressed that the $\mathrm{R}$ algorithm used to update the configurations is not exact and should have its accuracy tested carefully for each different value of the quark mass used, comparing when possible to simulations using the exact RHMC algorithm. Indeed, recent studies try to establish to what extent the use of the R algorithm may have influenced currently accepted results from lattice-QCD simulations. One study of this type considers possible systematic effects on results for the finite-temperature phase transition in the case of a realistic mass spectrum [18]. (Note that the transition in this case is not of second order.) It is generally believed that such systematic errors are negligible, or of the order of the statistical uncertainties in the simulations [9], but a detailed comparison must be made. We are currently extending our study using the RHMC algorithm.

As discussed here, the phase transition for two degenerate quark flavors is clearly especially well-suited for studying the effects of systematic errors in lattice simulations, since very large systematic effects would not be unexpected around a second-order phase transition. In fact, this case may be a more stringent test of the several possible systematic errors, such as discretization effects, errors associated with the updating algorithm or with the choice of the fermion discretization used in the simulations. 


\section{Acknowledgements}

This work was partially supported by FAPESP and CNPq.

\section{References}

[1] U. M. Heller, Recent progress in finite temperature lattice $Q C D, P o S$ LAT2006, (2006) 011, arXiv:hep-lat/0610114.

[2] Remarks On The Chiral Phase Transition In Chromodynamics, R. Pisarski, and F. Wilczek, Phys. Rev. D29 (1984) 338.

[3] F. Wilczek, Opportunities, challenges, and fantasies in lattice QCD, Nucl. Phys. Proc. Suppl. 119 (2003) 3.

[4] S. Ejiri, Lattice QCD thermodynamics with Wilson quarks, arXiv:hep-lat/0704.3747.

[5] F. Karsch, and E. Laermann, Susceptibilities, the specific heat and a cumulant in two flavor QCD, Phys. Rev. D50 (1994) 6954.

[6] S. Aoki et al. (JLQCD Collaboration), Scaling study of the two-flavor chiral phase transition with the Kogut-Susskind quark action in lattice QCD, Phys. Rev. D57 (1998) 3910.

[7] C. Bernard et al. (MILC Collaboration), Critical behavior in $N(t)=4$ staggered fermion thermodynamics, Phys. Rev. D61 (2000) 054503.

[8] M. D'Elia, A. Di Giacomo and C. Pica, Two flavor QCD and confinement, Phys. Rev. D72 (2005) 114510.

[9] G. Cossu, M. D’Elia, A. Di Giacomo and C. Pica, Two flavor QCD and confinement - II, arXiv:hep-lat/0706.4470.

[10] J. B. Kogut and D. K. Sinclair, Evidence for $O(2)$ universality at the finite temperature transition for lattice QCD with 2 flavours of massless staggered quarks, Phys. Rev. D73 (2006) 074512.

[11] E. Laermann, Chiral transition in 2 flavor staggered QCD, Nucl. Phys. Proc. Suppl. 60A (1998) 180.

[12] T. Mendes, Search for universal scaling at the chiral phase transition in 2-flavor lattice QCD, AIP Conf. Proc. 756 (2005) 413.

[13] T. Mendes, Lattice results for the QCD phase transition, Braz. J. Phys. 37 (2007) 597, arXiv:hep-lat/0609035.

[14] J. Engels, and T. Mendes, Goldstone-mode effects and scaling function for the three-dimensional $O(4)$ model, Nucl. Phys. B572 (2000) 289.

[15] J. Engels, S. Holtmann, T. Mendes, and T. Schulze, Finite-size-scaling functions for $3 d O(4)$ and $O(2)$ spin models and QCD, Phys. Lett. B514 (2001) 299.

[16] A. Cucchieri and T. Mendes, Equation of state for spin systems with Goldstone bosons: The 3d O(4) case, J. Phys. A38 (2005) 4561.

[17] S. R. Sharpe, Rooted staggered fermions: Good, bad or ugly?, PoS LAT2006 (2006) 022, arXiv:hep-lat/0610094.

[18] M. Cheng et al., Study of the finite temperature transition in 3-flavor QCD using the R and RHMC algorithms, Phys. Rev. D75 (2007) 034506. 